\title{
Binary Artificial Bee Colony Optimization for GENCOs' Profit Maximization under Pool Electricity Market
}

\author{
Manasarani Mandala \\ Electrical Engineering Department, \\ Indian Institute of Technology, Roorkee (IITR) \\ Roorkee, India
}

\author{
C.P.Gupta, Ph.D \\ Electrical Engineering Department, \\ Indian Institute of Technology, Roorkee (IITR), \\ Roorkee, India
}

\begin{abstract}
This paper proposes GENCOs' profit maximization using Binary Article Bee Colony Optimization based on global best parameters (GbBABC). The optimal rival bidding strategy is employed to maximize GENCOs' profit. Monte Carlo (MC) simulation has been used to predict the bidding behavior of the rivals. In this paper, a bi-level optimization problem has been proposed to obtain the optimal bidding strategy of a supplier in which lower level problem represents the market clearing process of the system operator (SO) and the upper level optimization problem represents the supplier's profit maximization function, which is a non-linear function. In the proposed algorithm, global best parameter was incorporated into $\mathrm{BABC}$ algorithm, which makes the exploitation capacity improved and convergence speed quickened. At the same time, in order to maintain the population diversity the bit mutation operator is also performed. The feasibility of the proposed approach is analyzed on IEEE 30-bus system and IEEE-57 bus system. Results obtained using the GbBABC algorithm have been compared with those obtained using standard Artificial Bee Colony (ABC) optimization, global best guided $\mathrm{ABC}(\mathrm{GbABC})$ and global best distance guided $\mathrm{ABC}(\mathrm{GbdABC})$.
\end{abstract}

\section{Keywords}

GENCOs' profit maximization; optimal bidding strategy; Market Clearing Price; Binary Artificial Bee Colony Optimization; Artificial Bee Colony Optimization.

\section{INTRODUCTION}

Recent change in regulatory policies in electricity industries has created competitive environments and markets for power suppliers. Therefore, maximizing the profit, minimizing the risk and gaining the competitive advantage by suppliers are possible through strategic bidding [1]. For a generating company, it is very important to construct an optimal bidding strategy in order to maximize its profit. The main factors, which affect the bidding strategy decision of an individual supplier, are the demand variation, generator production cost, operating constraints or some regulatory constraints, other competitors bidding behavior, etc. Among them, the most uncertain factor is rival generators' bidding behavior that compounds the difficulties in bidding strategy decision process [1] due to special nature of electricity compared to the other commodities, where each player tries to play game to maximize their own profit. Recently, there are a number of researchers investigating optimal bidding strategies in locational marginal price (LMP) based markets. In Li et al., [2], a game-based bidding strategy for GenCos with incomplete information is proposed to find the supply function equilibrium. In non-cooperative game theory approach [3,4], strategic bidding problem was solved using Nash equilibrium.

With the complexity of strategic bidding problems, an efficient heuristic approach is needed to provide the optimal solution. Different techniques have been employed to develop optimal bidding strategy. Bajpai et al.[5] proposed optimal bidding strategy problem using PSO has in pool electricity market. Jain et.al.,[6] proposed an optimal bidding strategy considering risk constraints to provide profit maximization is solved using GA. Gountis et al.[7] introduced genetic algorithm (GA) and Monte Carlo (MC) simulation are applied to provide the optimal bidding strategy for maximizing the GENCOs' profit. Soleymani [8] introduced the a new enhanced method that uses the combination of particle swarm optimization (PSO) and simulated annealing (SA) to predict the bidding strategy of Generating Companies (Gencos) in an electricity market. Peng et al. [9] introduced Fuzzy adaptive Differential Evolution (FADE) is used to solve multi objective optimization problem with the consideration of maximal profit and minimal bidding risk and emissions.

Bajpai et al. [10] introduced A new method based on the optimal bidding strategy in a uniform price spot market using fuzzy adaptive particle swarm optimization (FAPSO), Monte Carlo (MC) method is used to simulate the rivals' behavior (Bajpai et al. 2007).The simulation results indicate that FAPSO is superior to GA in both searching performance and execution time. However, a proper design of the fuzzy set is needed to adapt the velocity weight for the particular problem. These methods have the advantage of searching the solution space more thoroughly, but have limitations of their sensitivity to the choice of parameters such as the crossover and mutation probabilities, instable convergence, slow and easy to premature exist in GA, temperature in SA, scaling factor in EP and inertia weight and learning factors in PSO. The Artificial Bee Colony technique can generate better optimal solution in less calculation time with stable convergence characteristic compared to other populationbased methods.

Karaboga introduced a bee swarm algorithm called the Artificial Bee Colony (ABC) algorithm that simulates the foraging behavior of bees [11] for multimodal and multidimensional numerical optimization problems. Due to its simplicity and ease of implementation, the $\mathrm{ABC}$ algorithm has captured much attention and has been applied to solve many engineering optimization problems [12-21]. Karaboga [12] proposed a new method based on the ABC algorithm was used for designing digital infinite impulse response (IIR) filters, and its performance was compared with those of other techniques. Basu [13] applied the ABC algorithm to economic dispatch problem considering multi area criterion. Singh [14] used the $\mathrm{ABC}$ algorithm for leaf-constrained minimum 
spanning tree problem and compared the performance of the $\mathrm{ABC}$ against the best approaches proposed in the literature. Tasgetiren et al. [15] developed a discrete model of the ABC and applied it to total flow-time minimization in permutation flow shops and it was used for the lot-streaming flow shop scheduling problem. Ma et al. [16] used the ABC for synthetic aperture radar (SAR) images segmentation. Huang et al. [17] proposed $\mathrm{ABC}$ for fault section estimation in power systems. To make $\mathrm{ABC}$ more effective, in certain applications, the basic $\mathrm{ABC}$ is combined with other evolutionary optimization algorithms to form hybrid $\mathrm{ABC}$ algorithm. Liao et al.[18] proposed a new approach, an adaptive artificial bee colony algorithm for long-term economic dispatch in cascaded hydropower systems. Narayana et al.[19] proposed binary/real coded artificial bee colony algorithm for thermal unit commitment problem. Bin et al.[20] introduced a hybrid artificial bee colony assisted differential evolution algorithm for optimal reactive power flow problem. Ozyon et al.,[21] introduced incremental artificial bee colony with local search (IABC) is another variant of $\mathrm{ABC}$ to solve economic dispatch problem.

In the $\mathrm{ABC}$ algorithm, there are three types of (artificial) bees, namely employed bees, onlooker bees, and scout bees. Each employed bee is associated to a different solution of the optimization problem to be solved. At each algorithm iteration, an employed bee explores the neighborhood of the solution to which it is associated. Onlooker bees also explore the neighborhood of solutions; however, differently from employed bees, they are not associated to one fixed solution, but they choose the solution they explore in iteration probabilistically as a function of a solution quality. If the neighborhood of a solution has been explored unsuccessfully for a given number of times, a new solution in the search space is chosen uniformly at random by a scout bee, adding an exploration feature to the algorithm. The $\mathrm{ABC}$ algorithm can be seen as a population based local search algorithm, where at each iteration the neighborhood of each of the solutions in the population is explored. It produces the new solution according to the stochastic variance process. In this process, the magnitudes of the perturbation are important since it can affect the new solution. The convergence speed of $\mathrm{ABC}$ is slow towards high quality solutions because of its stochastic nature, so it can easily get trapped in the local optimum. Such a position update strategy is good at exploration, however, is poor at exploitation hence convergence speed [22]. To improve the performance of $\mathrm{ABC}$ some researchers proposed many improved strategies. Alatas et al. [23] introduced the chaotic maps into the initialization and chaotic search into the scout bee phase and proposed the chaotic ABC algorithm. Gao and Liu [24] also employed both the chaotic systems and opposition-based learning methods to enhance the global convergence. But the chaotic base $\mathrm{ABC}$ algorithm focuses on avoiding the local optima, but brings in more extra function evaluations in chaotic search as a result it affects on convergence speed. Zhu et al. [25] proposed Gbest guided $\mathrm{ABC}$ algorithm by incorporating the information of the global best solution into the solution search equation to improve the exploitation. Banharnsakun et al. [26] introduced a best-so-far method for solution updates in the $\mathrm{ABC}$ algorithm, and the searching method based on a dynamic adjustment of search range depending on the iteration was also introduced for scout bees. In spite of these improvements, $\mathrm{ABC}$ may sometimes fail to progress in the direction of the global optimal for multimodal nonlinear functions. So, the proposed approach introduces Binary $\mathrm{ABC}$ based on global parameters (GbBABC). In the proposed algorithm, global best parameter was incorporated into $\mathrm{BABC}$ algorithm, which makes the exploitation capacity improved and convergence speed quickened. At the same time, in order to maintain the population diversity, the bit mutation operator was accepted for the proposed algorithm.

The main contributions of this paper are as follows:

i. Developing optimal bidding strategy for GENCOs profit maximization in competitive electricity market is modeled as a bi-level optimization problem in which the upper sub-problem is representing maximization of GENCOs' profit and the lower sub-problem representing the market clearing price. GENCOs' profit maximization is performed based on optimal rival bidding strategy.

ii. Novel technique Binary Artificial Bee Colony $(G b \mathrm{BABC})$ algorithm based on global parameters is used to implement the proposed approach for GENCOs' profit maximization under pool electricity market.

The remaining sections of this paper are organized as follows: Overview of the Artificial Bee Colony (ABC) is presented in section 2. The proposed GbBABC technique for optimal bidding strategy is presented in Section 3. Mathematical formulation of GENCOs' profit maximization is discussed in Section 4.Numerical results are presented and discussed in Section 5. Finally, conclusion is given in Section 6. So, next section is dealing with the Overview of the Artificial Bee Colony $(\mathrm{ABC}$.

\section{OVERVIEW OF THE ARTIFICIAL BEE COLONY OPTIMIZATION}

The artificial bee colony algorithm is a novel swarm intelligence algorithm and first proposed by Karaboga [11] in 2005. It simulates the behavior of honey bee foraging foods. In the $\mathrm{ABC}$ algorithm, artificial bees can be divided into three groups, i.e., employed bees, onlooker bees, and scout bee. A bee going to the food source itself is named as an employed bee, a bee waiting in the dance area and choosing a food source is called as an onlooker bee, a bee carrying out random search is named as a scout bee. A half of artificial bees are employed bees and the other half of bees are onlooker bees. The number of employed bees in colony is equal to the number of food sources. For this reason, to every food source, there is only one corresponding employed bee. The employed bee whose food source is exhausted by the employed and onlooker bees becomes a scout [23]. In the original $\mathrm{ABC}$ algorithm, there is only one scout bee in bee colony. The ABC algorithm has three parts including the employed phase, the onlooker phase, and the scout phase. The main steps of the $\mathrm{ABC}$ algorithm are as follows:

1. Initialize the population.

2. Send the employed bees to the food sources.

3. Memory the best food source in employed bees by fitness evaluation.

4. Employed bees come back to hive and share information of food sources with onlooker bees, then onlooker bees fly to the food sources which they have chosen.

5. Memory the best food source in onlooker bees by fitness evaluation. 
6. The scout bees fly to the search area and look for new food sources.

7. While the terminal condition is met or maximum cycle number is reached, Algorithm stop. Otherwise, go back to step 2.

In the initialization step, employed bees selected a set of food source positions and calculate their nectar amounts. Then, every employed bee chooses a new food source by means of nectar amount information in the neighborhood of the present one. After that, employed bees return to the hive and share the information of the food sources with the onlooker bees waiting on the dance area. Onlooker bees choose the food source determined by a probability value. In the scouts phase, scout bee replaces the food source which has not been selected by the artificial bees. The $\mathrm{ABC}$ algorithm consists of four phases: initialization, employed bee, onlooker bee and scout bee and implementation procedure is explained below.

\section{i. $\quad$ The Initial Population of Solutions:}

$\mathrm{ABC}$ searches for a global optimum point in a D-D real parameter space $\mathfrak{R}^{D}$ by initializing a random population composed of D-D real valued parameter vectors. These vectors, also called food sources, forms trial solutions to the multidimensional problem. The components of the food sources are modified over subsequent iteration called cycles. The subsequent generations are indicated by $C=0,1, \ldots, M C N$ (maximum cycle number). The following notation maybe adopted for denoting the $i^{\text {th }}$ food source at the current cycle:

$$
\overrightarrow{x_{i}^{C}}=\left[x_{i, 1}^{C}, x_{i, 2}^{C}, x_{i, 3}^{C}, \ldots \ldots, x_{i, \mathrm{D}}^{C}\right]
$$

Each parameter of the problem must have its value restricted within a certain range since these are related with physical measures having natural bounds. The initialization of food source (at $\mathrm{C}=0$ ) must ensure that they are distributed across the solution space and cover the search range as much as possible. The common practice is to use uniform initialization on a component basis as,

$$
x_{i j}=x_{j \min }+\operatorname{rand}_{1}(0,1)\left(x_{j \max }-x_{j \min }\right)
$$

where $j=1, \ldots . . D, i=1, \ldots . ., S N$ for a D-dimensional problem employing $S N$ food sources (employed bees) and $\operatorname{rand}_{j}(0,1)$ is a uniformly distributed number lying between $\left(0 \leq \operatorname{rand}_{j}(0,1) \leq 1\right)$. The solution space bounds $\vec{x}_{\text {max }}=\left(x_{1, \text { max }}, x_{2, \text { max }}, \ldots ., x_{\mathrm{D}, \max }\right)$ and $\vec{x}_{\text {min }}=\left(x_{1, \text { min }}, x_{2, \text { min }}, \ldots ., x_{\mathrm{D}, \text { min }}\right)$. are predefined. Following the initialization process, the objective functional value (specific to the problem) of all the food sources is evaluated $f_{i}=F\left(x_{i}^{C}\right)$. Additionally, based on our objective (to maximize or to minimize), fitness value of the food sources is calculated as:

$$
\text { fitness }_{i}= \begin{cases}1 /\left(1+f_{i}\right) & \text { if } f_{i} \geq 0 \\ 1+a b s\left(f_{i}\right) & \text { if } f_{i}<0\end{cases}
$$

where $f_{i}$ is the actual objective (or cost) function to be optimized. The formula for the fitness function satisfies the minimization condition. For maximization purpose we can directly set fitness $s_{i}$ equal to $f_{i}$ the trial counter of each food source is reset to 0 . The termination criterion is chosen based on the requirement of the user. The process can end when the value of $\mathrm{C}$ reaches the maximum cycle number $M C N$ or the error value attained is less than the predefined accuracy threshold or, as in our case, a given number of function evaluations (FEs) have been expended.

\section{ii. $\quad$ The Search Phase of Employed Bees}

In this phase the employed foragers seek a food source with the intention of exploiting it. It is a customary scheme to set only one forager for a given food source such that bijective mapping occurs. The forager exploits the local site and modifies it position around the source by changing a single positional parameter as,

$$
v_{i j}^{C}=x_{i j}^{C}+\phi_{i j} \times\left(x_{i j}^{C}-x_{k j}^{C}\right)
$$

where $i$ is the running index and $\mathrm{k}$ can take any values except $i$, i.e., $(k \in\{1,2, \ldots, S N\} \backslash\{i\})$ with $\phi_{i j}$ is an uniformly distributed random number sampled from the range $[-1,1]$ and determines the degree of positional perturbation of the forager. In cases of trial solutions overshooting the bounds they are reinitialized within the bounds. While executing the local fly-by motion around the food source, the foragers continually analyze its near vicinity in search of better food source. If a food source of higher fitness is found, then greedy selection occurs in order to replace the present position $\overrightarrow{x_{i}^{C}}$ of its employed food source by the newly found one $\overrightarrow{v_{i}^{C}}$ and the corresponding trial counter is reset. This is analogous to the forager memorizing the new food source when it has better nectar content (fitness value). It is unlikely to visit the food source again. This saves additional computational burden. However in case of no improvement in fitness value, the trial counter is incremented by 1 to indicate worsening fitness value. As can be seen from Eq. (3), as the difference between the parameters of the $x_{i j}$ and $x_{k j}$ decreases, the perturbation on the position $x_{i j}$ decreases. Thus, as the search approaches to the optimal solution in the search space, the step length is adaptively reduced. If a parameter value produced by this operation exceeds its predetermined boundaries, the parameter can be set to an acceptable value. In this work, the value of the parameter exceeding its boundary is set to its boundaries is defined as,

$$
x_{i}= \begin{cases}x_{i}=x_{i \max } & \text { if } x_{i}>x_{i \max } \\ x_{i}=x_{i \text { min }} & \text { if } x_{i}>x_{i \text { min }}\end{cases}
$$

\section{iii. $\quad$ The Selection Phase of Onlooker Bees}

The nectar collecting foragers after loading nectar travels back to the hive to unload it. In the process it may communicate the information about newly found sources to the waiting onlooker in the hive via waggle dance. The purpose is to convey the distance, direction and nectar content of their present food source. Depending on the fitness value conveyed by the waggle dancers, the onlookers select a single food site. The selection is performed by roulette wheel process where the higher fitness food sources have more chances of being selected than less fit one. The probability of get-ting selected (each slice) is proportional to fitness value and is given by,

$$
p_{i}=\frac{\text { fitness }_{i}}{\sum_{i=1}^{S N} \text { fitness }_{i}}
$$


The $i^{\text {th }}$ food source is selected if a randomly generated number within the range $(0,1)$ is less than the probability $p_{i}$. If the onlooker had already visited the site it can relocate it easily or else it moves following the direction. Again a similar positional modification as in Eq. (4) occurs followed by greedy selection and trial counter update. This process is continued until all the onlookers have been allotted food sources. But one of the drawbacks of this process is its primary focus on fitness for food source selection, ignoring the $T / E$ ratio (total reward obtained $\mathrm{T}$ to the Euclidean distance $\mathrm{E}$ as measured from the hive) which is the fundamental decision making criteria for food source selection in bee colonies. Karaboga [30] stressed on the importance of $T / E$ ration. The biological motivation behind the $T / E$ ratio is that the bees must be sufficiently rewarded while travelling to a distant site. The reward is in the form of nectar content (fitness value) compensating the effort undertaken to perform the journey.

\section{iv. The scout bee phase}

It is natural for a food source to become exhausted after being repeatedly exploited by the foragers. So to save wastage of energy, the foragers avoid making further travels to a food source when it is deemed to be exhausted. The employed bee abandons the food source and behaves as scout bee that performs random walks in the search space to locate a new food source. This analogy is mapped by setting a parameter limit. There occurs an instant when trial counter, which is incremented by 1 each time the forager is unable to improve upon its previous value, reaches its limiting value. In such a case, it is thought to be exhausted and the food source is reinitialized randomly and the corresponding trial counter is reset to 0 .The parameter limit significantly affects the performance of the $\mathrm{ABC}$ algorithm. Too small a value is identical to initializing food sources prematurely while a considerably larger value amounts to wastage of useful FEs. The suitable value of limit is problem dependent and Karaboga [22] suggested that the value of limit be set to SN.D. The greedy selection scheme and the random selection scheme increases the local and global search capability of the algorithm [11]. The ABC algorithm is an iterative algorithm and maximum iteration or function evaluation number or an error rate can be used as the stopping criterion for the algorithm. In the basic ABC algorithm, numbers of the food sources, employed bees and onlooker bees are equal to each other. As mentioned above, the standard $\mathrm{ABC}$ has limitation of convergence speed due to its stochastic nature. So, it can easily get trapped in the local optimum. So, to improve the global search capability and convergence speed the new food source search is directed and guided with global best.

\section{PROPOSED BINARY ARTIFICIAL BEE COLONY ALGORITHM (BABC)}

The original $\mathrm{ABC}$ algorithm is mainly used for continuous optimization, however many engineering practices have been described as a combinatorial optimization problem. Binary artificial bee colony (BABC) algorithm can be used to optimize the 0-1 integer programming problem. Kashan et al.,[27] proposed DisABC algorithm in which avoids converting continuous domain to the discrete domain, it helps reducing complexity. Based on this concept the proposed approach introduced Binary $\mathrm{ABC}$ based on global parameters. In the proposed algorithm, global best parameter was incorporated into BABC algorithm, which makes the exploitation capacity improved and convergence speed quickened. At the same time, in order to maintain the population diversity, the bit mutation operator was accepted for the proposed algorithm. Generally, in population-based optimization methods, it is desirable to encourage the individuals to wander through the entire search space, without clustering around local optima, during the early stages of the optimization. On the other hand, during the latter stages, it is important to enhance convergence toward the global optima, to find the optimal solution efficiently. So, the proper control of global exploration and local exploitation is required in finding the optimum solution efficiently.

The basic ABC algorithm does not use the global best solution of population in each generation to direct the search trajectory towards global optimal solution. It has been reported in old literature that the $\mathrm{ABC}$ algorithm has good global search capability but poor local search capability and hence convergence speed. In order to enhance the exploitation capability of $\mathrm{ABC}$ algorithm i.e., to improve the ability to apply the knowledge of the previous good solutions to find even better candidate solutions. An analysis of the studies on the $\mathrm{ABC}$ in the literature indicates that the $\mathrm{ABC}$ has powerful exploration ability but poor exploitation ability hence slower convergence speed. The performance of $\mathrm{ABC}$ is depending on new food search process i.e., search phase of employed bees. So, to provide powerful global search capability and to improve the convergence speed, now the $\mathrm{ABC}$ search phase of employed bees is guided with global best (Gbest) value to find new food source search process. Andrej et al., [28] proposed Gbest guided $\mathrm{ABC}(\mathrm{GbABC})$ algorithm by incorporating the information of the global best solution into the solution search equation to improve the exploitation. This modification is inspired by the usage of the global-best solution to influence particles in particle swarm optimization. According to this approach the modified Eq. (5) directs the search path towards global optima,

$$
v_{i j}=x_{i j}+\phi_{i j}\left(x_{i j}-x_{k j}\right)+\psi_{i j}\left(x_{g b e s t j}-x_{i j}\right), \quad i \neq k
$$

where $x_{g b e s t j}$ is the $j^{\text {th }}$ coordinate of the global-best solution, and $\psi_{i j}$ is a random number drawn uniformly at random in $[0, C]$, where $C$ is a non-negative number which is tuning parameter and it is set as 1.5[28]. Further authors [28] introduced the concept of Gbest distance based $\mathrm{ABC}$ $(G b d A B C)$. Contrary to the basic approach of calculating the probability of food site selection based on the fitness value, as in Eq. (2), Authors computed probability based on the distance between two best solutions. The modification is that it is more probable to find a better solution by searching between two good solutions that are close to each other in the solution space, which improves the convergence speed. It helps in controlling premature convergence by preventing overcrowding of forager population around the best-so-far population. To further strengthen the exploitation capability of $\mathrm{ABC}$ algorithm, Eq. (4) is modified to obtain the following Eq. (6) which provides the better solution quality in the search space.

$$
p_{k}=\frac{1 / d\left(x_{i}, x_{k}\right)}{\sum_{i=1, l \neq i}^{S N} 1 / d\left(x_{i}, x_{l}\right)}
$$

where $d\left(x_{i}, x_{k}\right)$ is the Euclidean distance between two solutions $x_{i}$ and $x_{k}$. This is the distance between the current 
position and a potential reference employed bees influences the selection probability. Therefore, instead of using the same probability for all reference employed bees, an employed bees at position $p i$ chooses the reference employed bees where $k \in\left\{1, \ldots \ldots, n_{e b}\right\}$ with $k \neq i . n_{\mathrm{eb}}$ is the new employed bees. Thus, the closer a solution is to $x_{i}$, the higher is the probability of choosing it. Thus the new search process takes advantage of knowledge of the global best solution to modify the search trajectory similar to PSO. The tuning parameter $C$ should be properly chosen so that the new solution is not driven away from desired candidate solution. The small value of this control parameter may not improve solution quality as (5) and (6) produces nearly same results. On the other hand, the large value of $\mathrm{C}$ may reduce exploration capability of search process. So, here it is set as 1.5 [28].

\section{Binary $A B C$ guided with Gbest (BGbABC)}

This procedure directly encodes the problems in discrete space, and modifies the food source generating method of continuous $\mathrm{ABC}$ algorithm. The bit operation replaces the traditional vector calculation. Here new food source searching formula is guided with Gbest (GbABC). The ABC algorithm is essentially a real-coded algorithm, thus some revisions are needed to enable it to deal with the binary-coded optimization problem. In the proposed binary $\mathrm{ABC}$, the relevant variables are interpreted in terms of changes of probabilities. In binary $\mathrm{ABC}, x_{i j}$ and $x_{k j}$ take values of 0 or 1 and $\phi_{i j}$ is a random number generated in the range $[-1,1]$. When the position is modified in the binary mode, the modified position, $v_{i j}$, will vary between $[0,1]$. If $v_{i j}$ is higher, the individual is more likely to choose a 1 otherwise it chooses 0 . The threshold level can be made to range from 0 to 1 . To accomplish this, the proposed function can be written as,

$$
\begin{aligned}
& f\left(v_{i j}\right)=\tanh \left(\left|v_{i j}\right|\right)=\frac{\exp \left(2 *\left|v_{i j}\right|-1\right)}{\exp \left(2 *\left|v_{i j}\right|+1\right)} \\
& v_{i j}^{\prime}= \begin{cases}1, & \text { if } f\left(v_{i j}\right)>0, \\
0, & \text { otherwise }\end{cases}
\end{aligned}
$$

If the output of the generated function is a positive value, the corresponding $v_{i j}^{\prime}$ is recorded as 1 ; otherwise, it is recorded as 0 .In order to prevent the algorithm stagnation and ensure the new food source changed at least one dimension, a certain bit of the new food source does mutation operation, namely,

$$
v_{i j}^{\prime}= \begin{cases}1-v_{i j}^{\prime}, & \text { if } j=\operatorname{rand} 2, \\ 0, & \text { otherwise }\end{cases}
$$

where, rand 2 is the random integer between $[1, \mathrm{D}]$ and rand $2 \neq$ rand 1 . So, Next section is dealing with the problem of GENCOs' profit maximization.

\section{MAXIMIZATION OF GENCOS' PROFIT}

In competitive environment, several participants compete to win a share of the market and bid against each other. Each participant submits a sealed bid to the power exchange. The Independent System Operator (ISO) decides the Market Clearing Price (MCP) by matching the aggregate demand to the aggregate supply. Then the system operator checks system security and reliability to ensure that the resulting bidding schedule is feasible. In a perfect power market, action of single supplier cannot influence the market price. But most of the electricity markets are not perfectly competitive. As a result, a supplier can increase its profit by strategic bidding, or in other words, through exercising market power. As a result, optimal bidding strategy is to be adopted by participating GENCOs' for the maximization of their own profit. The strategic bidding is modeled as bi level optimization problem, in which upper level optimization problem represents the profit maximization for the generator- $j$ and the lower level optimization problem represents the market clearing. Consider a system consist of ' $\mathrm{m}$ ' suppliers participating in a pool-based single-buyer electricity market in which the sealed auction with a uniform market clearing price (MCP) is employed. The difference between the market clearing price and the actual cost of the GENCOs' will reflects the profit of supplier- $i$ $(i=1,2, \ldots \ldots \mathrm{m})$ is described as,

$$
\begin{aligned}
& \text { Profit }_{i}=\Pi_{j}=M C P \times P_{i}-C_{i c}\left(P_{i}\right) \\
& \text { Total Profit }=\sum_{i=1}^{m} \Pi_{i}=\sum_{i=1}^{m} \text { Profit }_{i}
\end{aligned}
$$

Subject to,

Power generation limits:

$$
\sum_{i=1}^{m} P_{i}=P_{d}(\lambda)
$$

Power balance constraints:

$$
P_{\min , i} \leq P_{i} \leq P_{\max , i}, \quad i=1,2, \ldots \ldots . . m
$$

where, MCP is market clearing price to be estimated and it is also referred as $\lambda . \mathrm{P}_{i}$ is the active power output of the $i^{\text {th }}$ supplier. $P_{\min , i}$ and $P_{\max , i}$ are the generation output limits of the $i^{\text {th }}$ supplier. $P_{d}(\lambda)$ is the aggregate pool load forecast. $C_{\mathrm{i}}$ is the cost coefficient of the $i^{\text {th }}$ supplier. Assume that each supplier is required to bid a linear supply function to the pool. The aggregate pool load forecast as follows is,

$$
P_{d}(\lambda)=P_{d 0}-K \lambda
$$

where $P_{d 0}$ is a constant number and $\mathrm{K}$ is a non-negative constant used to represent the load price elasticity.

Estimation of Rival Bidding Strategy

In competitive environment, several participants compete to win a share of the market and bid against each other. Each participant submits a sealed bid to the power exchange.

$$
K_{i}=\frac{a_{i}}{a_{i c}}=\frac{b_{i}}{b_{i c}}=\frac{c_{i}}{c_{i c}}
$$

Where $a_{i}, b_{i}$ are the bidding coefficients and $a_{i c}, b_{i c}, c_{i c}$ are the actual coefficients of generators. The bidding parameter $K_{\mathrm{i}}$ represents the marginal cost of Genco.

The actual cost is of the form,

$$
C_{i c}\left(P_{i}\right)=\frac{1}{2} a_{i c} P_{i}^{2}+b_{i c} P_{i}+c_{i c}
$$

The bid coefficients have the following relationship with the cost coefficients,

$$
M C P_{i}=b_{i}+a_{i} P_{i}
$$




$$
\max \sum_{i=1}^{N_{G}} M C P_{i}
$$

For a fixed demand $P_{d 0}$, the supply curve of bidder- $i$ can be written as,

$$
\sum_{i=1}^{N_{g}} \boldsymbol{P}_{i}=\sum_{i=1}^{N_{g}} \frac{M C P}{K_{s i}}-\sum_{i=1}^{N_{g}} \frac{b_{i}}{K_{s i}}
$$

Where $K_{s i}=K_{i} \bullet a_{i c}$ is the bidding slope of supplier- $i$.

MCP will be obtained by solving the following equation

$$
M C P=\left(P_{D 0}+\sum_{i=1}^{N_{g}} \frac{b_{i}}{K_{s i}}\right) /\left(\sum_{i=1}^{N_{g}} \frac{1}{K_{s i}}\right)
$$

A function $\mathrm{m}_{\mathrm{si}}\left(\mathrm{m}_{\mathrm{R}}\right)$ derived from rivals' aggregated bidding response, is derived utilizing the equation (18) as following.

$$
K_{s i \max }\left(m_{R}\right)=\frac{\left(\left(P_{D 0}+\sum_{i=1}^{N_{g}} \frac{b_{i}}{K_{s i}}\right) / P_{i}+\frac{b_{i c}}{a_{i c}}\right)-1}{\sum_{i=2}^{N_{g}} \frac{1}{K_{s i}}}
$$

where $m_{R}$ is the rival's bidding slope. For strategic bidding problems, Monte-Carlo (MC) approach is employed to simulate rivals' bidding behavior under which the uncertainty, arising due to bidding behaviors of market participants is represented by the distribution of historical bidding behaviors. Here, bidding estimation for each GENCO- $i$ with respect to rival $-j$ is computed using Gaussian distribution as follows:

$$
\operatorname{MCP}\left(p_{j}^{n}(t)\right)=\frac{1}{\sqrt{2 \Pi \sigma_{j}^{n}(t)}} \mathrm{e}^{-\frac{1}{2}\left(\frac{p_{j}^{n}-\mu_{j}^{n}(t)}{\sigma_{j}^{n}(t)}\right)^{2}}
$$

Here $\mu_{j}^{n}(t)$ and $\sigma_{j}^{n}(t)$ is the mean value and the standard deviation of the hourly forecasted MCP at time t, respectively. The rival's bid slope $K_{s i}\left(m_{R}\right)$ range falls between $[\mu(t)+3 \sigma(t)$, $\mu(t)-3 \sigma(t)]$ with probability 0.95 . The proposed problem is solved using GbBABC. So, Next section is dealing with Results and discussion.

\section{RESULTS AND DISCUSSION}

\subsection{IEEE 30-Bus System}

The system data is taken from [29]. This system consists of six GENCOs' who supply electricity to aggregate load and 41 lines. $\boldsymbol{P}_{d 0}$ is $283.4 \mathrm{MW}$ with inelastic load $(\mathrm{K}=0)$, considered for aggregated demand. To analyze the proposed approach, rival bidding strategy is employed to estimate the MCP using MC simulation with $(G b B A B C)$, then maximization of GENCOs' profit is performed using GbBABC. When the bidding process is over, ISO checks the system security to ensure whether the resulting bidding schedule is feasible. To show the effectiveness of the proposed approach, obtained results are compared with, standard $\mathrm{ABC}$ [11], Gbest guided ABC $(G b A B C)$ [28], and Gbest distance based ABC $(G b d A B C)$ [28]. According to the proposed approach firstly bidding strategies are estimated using $\mathrm{MC}$ simulation with GbBABC is listed in Table I and compared with the reported

\begin{tabular}{|c|c|c|c|c|c|}
\hline & $\begin{array}{c}\text { GENCO } \\
s^{\prime}\end{array}$ & $\begin{array}{r}A B C \\
{[11]}\end{array}$ & $\begin{array}{c}G b d A B C \\
{[28]}\end{array}$ & $\begin{array}{c}G b A B C \\
{[28]}\end{array}$ & $\begin{array}{c}\text { Propose } \\
\mathrm{d} \\
\mathrm{Gb} B A B \\
C\end{array}$ \\
\hline \multirow{6}{*}{$\begin{array}{c}\text { Optimal } \\
\text { bidding } \\
\text { (\$/MWh } \\
\text { r) }\end{array}$} & 1 & $\begin{array}{c}1.0232 \\
7\end{array}$ & 1.03632 & 1.04028 & 1.0533 \\
\hline & 2 & $\begin{array}{c}1.1565 \\
5\end{array}$ & 1.27082 & 1.31819 & 1.32219 \\
\hline & 5 & $\begin{array}{c}1.4002 \\
9\end{array}$ & 1.55505 & 1.76607 & 1.78607 \\
\hline & 8 & $\begin{array}{c}1.0305 \\
6\end{array}$ & 1.04567 & 1.07472 & 1.08272 \\
\hline & 11 & $\begin{array}{c}1.1290 \\
7\end{array}$ & 1.14560 & 1.18808 & 1.1980 \\
\hline & 13 & $\begin{array}{c}1.0706 \\
1\end{array}$ & 1.09359 & 1.11114 & 1.11214 \\
\hline \multirow{6}{*}{\begin{tabular}{|c|} 
Real \\
power \\
Marginal \\
Price \\
$(\lambda)$ \\
$(\$ / \mathrm{MWh}$ \\
$\mathrm{r})$ \\
\end{tabular}} & 1 & 17.59 & 19.40 & 20.39 & 21.46 \\
\hline & 2 & 16.77 & 18.62 & 20.30 & 20.47 \\
\hline & 5 & 24.77 & 27.01 & 29.99 & 30.14 \\
\hline & 8 & 25.79 & 28.05 & 30.26 & 31.32 \\
\hline & 11 & 26.89 & 29.92 & 32.11 & 33.93 \\
\hline & 13 & 26.57 & 28.90 & 31.20 & 33.22 \\
\hline
\end{tabular}
techniques. With these optimal bids, real power marginal prices are also computed and listed in Table I.
Table I: Optimal bidding and locational marginal price for IEEE-30 bus system

From the Table I it is observed that the proposed GbBABC is performed better than $\mathrm{ABC}, G b A B C$, and $G b d A B C$ method. Real power Marginal prices are also higher using GbBABC. Based on this bidding GENCOs' Profit maximization is

\begin{tabular}{|c|c|c|c|c|c|}
\hline & GENCOs' & $\begin{array}{r}A B C \\
{[11]}\end{array}$ & $\begin{array}{c}G b d A B C \\
{[28]}\end{array}$ & $\begin{array}{c}G b A B C \\
{[28]}\end{array}$ & $\begin{array}{l}\text { Proposed } \\
\text { GbBABC }\end{array}$ \\
\hline \multirow{6}{*}{$\begin{array}{c}\text { GENCOs' } \\
\text { Power } \\
(\mathrm{MW})\end{array}$} & G1 & 74.95 & 76.24 & 80.00 & 69.39 \\
\hline & $\mathrm{G} 2$ & 23.41 & 28.54 & 27.51 & 12.86 \\
\hline & G3 & 20.76 & 18.21 & 20.537 & 28.83 \\
\hline & G4 & 10.22 & 9.85 & 10.050 & 17.29 \\
\hline & G5 & 12.69 & 18.72 & 15.619 & 25.39 \\
\hline & G6 & 371.31 & 37.81 & 36.96 & 43.39 \\
\hline \multirow{6}{*}{$\begin{array}{l}\text { Profit } \\
(\$ / \mathrm{hr})\end{array}$} & 1 & \begin{tabular}{|l|}
488.385 \\
\end{tabular} & 519.707 & 549.117 & 605.927 \\
\hline & 2 & 163.161 & 175.1168 & 187.9326 & 236.5082 \\
\hline & 5 & 64.0788 & 92.7708 & \begin{tabular}{|l|}
81.1984 \\
\end{tabular} & 121.4915 \\
\hline & 8 & 207.4432 & 229.0100 & 241.3954 & 333.8458 \\
\hline & 11 & 99.9438 & 133.4178 & 148.0507 & 227.0781 \\
\hline & 13 & 101.5219 & 134.9959 & 187.0240 & 234.2271 \\
\hline \multicolumn{2}{|c|}{ Total Profit $(\$ / \mathrm{hr})$} & 1124.5 & 1285.0 & 1394.7 & 1759.1 \\
\hline \multicolumn{2}{|c|}{$\begin{array}{c}\text { Computation Time } \\
\text { (Secs) }\end{array}$} & 72 & 63 & 59 & 43 \\
\hline
\end{tabular}
performed and listed in Table II. And also corresponding power dispatch is also listed in Table II.

Table II: GENCOs' power dispatch and Profit for IEEE30 bus system

From the Table II it is observed that, power dispatched from all the GENCOs' using GbBABC is maximum compared to other reported techniques. For improving the profit of GENCOs', GbBABC is performed better than other reported techniques. So, In imperfect competitive electricity market with rival optimal bidding strategy GENCOs' improved their individual profit, hence total profit of GENCOs' is increased. The total profits of GENCOs' using all techniques are depicted in Fig.2. 


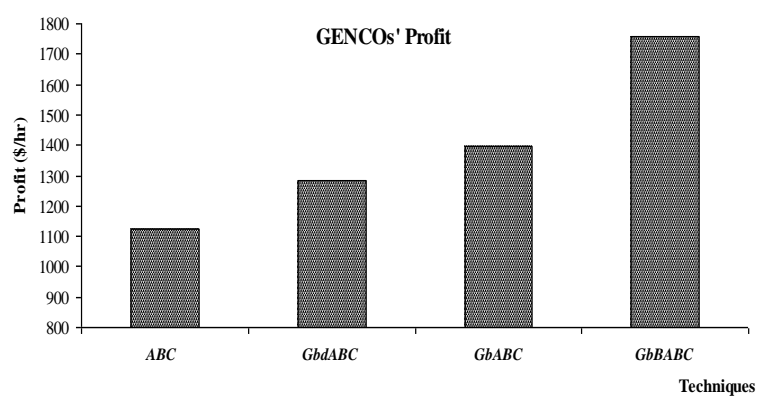

Fig.2. GENCOs' Profit for IEEE- 30 Bus System using all Techniques

From the Fig.2, it is observed that GbBABC is giving higher profit compared to other reported techniques. GENCOs' profit using $\mathrm{GbBABC}$ is $1759.1(\$ / \mathrm{hr})$ and it is reduced to 1394.7(\$/hr) using GbABC. The convergence criterion for GENCOs' profit is depicted in Fig.4. From the Fig.4 it is observed that, GbBABC finds better solution after 32 iterations, GbABC finds better solution after iteration 52 , GbdABC finds better solution after iteration 65, and $\mathrm{ABC}$ finds better solution after iteration 72. So, GbBABC converges faster than all the reported techniques. The time taken for the convergence of the proposed method is drastically reduced because of the global best parameter inclusion into $\mathrm{BABC}$ algorithm, which makes the exploitation capacity improved and convergence speed quickened. The bit mutation operator is performed, in order to maintain the population diversity.

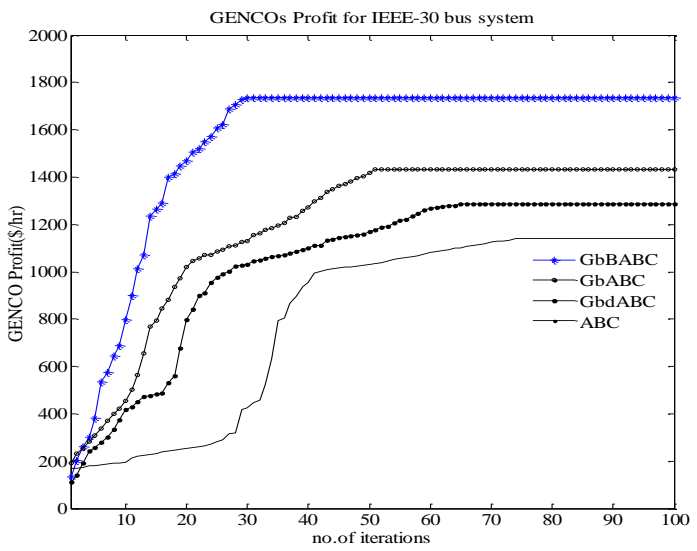

Fig.3.Convergence criterion for GENCOs' Profit for IEEE-30 bus system

Table III: GENCOs' optimal bidding and profit for IEEE57 bus system

\begin{tabular}{|c|c|c|c|c|c|}
\hline & GENCOs' & $\begin{array}{l}A B C \\
{[11]} \\
\end{array}$ & $\begin{array}{c}G b d A B C \\
{[28]}\end{array}$ & $\begin{array}{c}G b A B C \\
{[28]} \\
\end{array}$ & $\begin{array}{l}\text { Proposed } \\
\mathrm{Gb} B A B C\end{array}$ \\
\hline \multirow{7}{*}{$\begin{array}{c}\text { Optimal } \\
\text { bidding } \\
\text { (\$/MWhr) }\end{array}$} & 1 & 1.2105 & 1.2840 & 1.3523 & 1.3718 \\
\hline & 2 & 1.1507 & 1.1653 & 1.1698 & 1.1845 \\
\hline & 3 & 1.3005 & 1.4290 & 1.4823 & 1.4868 \\
\hline & 6 & 1.5746 & 1.7487 & 1.9859 & 2.0084 \\
\hline & 8 & 1.1589 & 1.1759 & 1.2085 & 1.2175 \\
\hline & 9 & 1.2696 & 1.2882 & 1.3360 & 1.3472 \\
\hline & 12 & 1.2039 & 1.2297 & 1.2494 & 1.2506 \\
\hline \multirow{6}{*}{$\begin{array}{c}\text { Real } \\
\text { power } \\
\text { Marginal } \\
\text { Price }(\lambda) \\
(\$ / \text { MWhr })\end{array}$} & 1 & 17.02 & 18.94 & 19.31 & 21.93 \\
\hline & 2 & 16.97 & 18.63 & 19.54 & 20.19 \\
\hline & 3 & 16.25 & 17.94 & 19.45 & 19.61 \\
\hline & 6 & 23.36 & 25.39 & 28.07 & 28.20 \\
\hline & 8 & 24.27 & 26.31 & 28.31 & 29.25 \\
\hline & 9 & 25.24 & 27.98 & 29.96 & 31.57 \\
\hline
\end{tabular}

\begin{tabular}{|c|c|c|c|c|c|}
\hline & 12 & 24.95 & 27.07 & 29.14 & 30.94 \\
\hline \multirow{4}{*}{$\begin{array}{c}\text { Profit } \\
(\$ / \mathrm{hr})\end{array}$} & 1 & 304.40 & 306.93 & 364.55 & 372.58 \\
\cline { 2 - 6 } & 2 & 101.46 & 102.31 & 121.51 & 124.19 \\
\cline { 2 - 6 } & 3 & 53.252 & 73.05 & 71.87 & 99.60 \\
\cline { 2 - 6 } & 8 & 172.39 & 180.33 & 213.68 & 273.711 \\
\cline { 2 - 6 } & 9 & 84.058 & 105.06 & 131.05 & 186.17 \\
\cline { 2 - 6 } & 12 & 135.59 & 137.89 & 166.35 & 193.90 \\
\hline Total Profit \$/hr) & 934.5 & 1011.9 & 1234.6 & 1442.2 \\
\hline $\begin{array}{c}\text { Computation Time } \\
\text { (Secs) }\end{array}$ & 70 & 67 & 63 & 46 \\
\hline
\end{tabular}

\section{IEEE-57 BUS SYSTEM}

The system data is taken from [29]. This system consists of seven GENCOs' who supply electricity to aggregate load and eighty transmission lines. $P_{d 0}$ is $325 \mathrm{MW}$ with inelastic load

$(\mathrm{K}=0)$, considered for aggregated demand. Similar procedure followed here. To analyze the proposed approach, rival bidding strategy is employed to estimate the MCP using MC simulation with $(G b B A B C)$, and then maximization of GENCOs' profit is performed using $G b \mathrm{BABC}$. When the bidding process is over, ISO checks the system security to ensure whether the resulting bidding schedule is feasible. To show the effectiveness of the proposed approach, obtained results are compared with, standard $\mathrm{ABC}$ [11], Gbest guided $\mathrm{ABC}(G b A B C)$ [28], and Gbest distance based ABC $(G b d A B C)$ [28]. According to the proposed approach firstly bidding strategies are estimated using MC simulation with GbBABC is listed in Table I and compared with the reported techniques. With these optimal bids, real power marginal prices and also GENCOs' profits are also computed and listed in Table III. From the Table III it is observed that, optimal bids for all the GENCOs' are maximum using GbBABC compared to other reported techniques. Hence GENCOs' individual profits are also improved using GbBABC. For improving the profit of GENCOs', GbBABC is performed better than other reported techniques. So, in imperfect competitive electricity market with rival optimal bidding strategy GENCOs' improved their individual profit, hence total profit of GENCOs' is increased. The total profits of GENCOs' using all techniques are depicted in Fig.4.

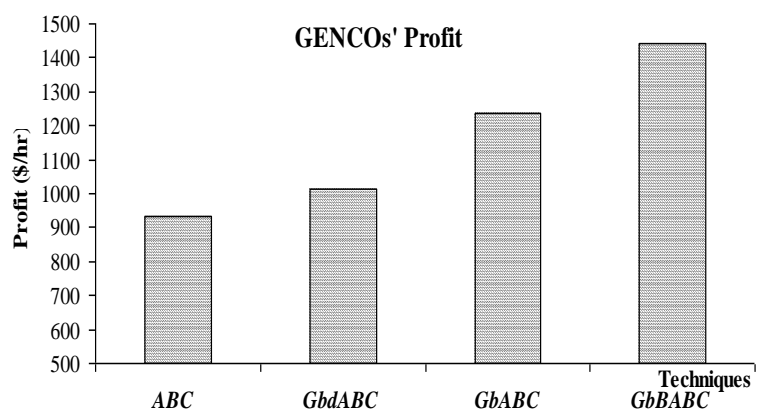

Fig.4. GENCOs' profit for IEEE- 57 bus system using all techniques

From the Fig.4, it is observed that GbBABC is giving higher profit compared to other reported techniques. The convergence criterion for GENCOs' profit is depicted in Fig.4. From the Fig. 4 it is observed that, GbBABC finds better solution after 29 iterations, GbABC finds better solution after iteration 49, BFO finds better solution after iteration 59, GbdABC finds better solution after iteration 65, and $\mathrm{ABC}$ finds better solution after iteration 74. So, GbBABC converges faster than all the reported techniques. The time taken for the convergence of the proposed method is drastically reduced because of the global best parameter 
inclusion into $\mathrm{BABC}$ algorithm, which makes the exploitation capacity improved and convergence speed quickened. The bit mutation operator is performed, in order to maintain the population diversity.

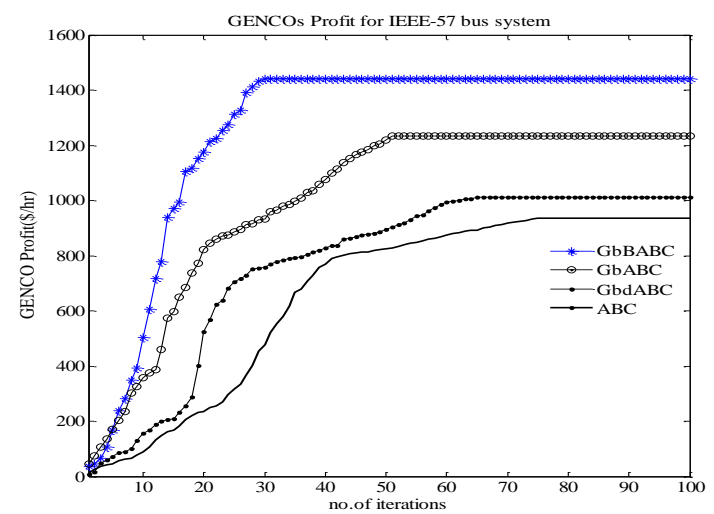

Fig.5.Convergence criterion for GENCOs' Profit for IEEE-57 bus system

\section{CONCLUSION}

This paper focuses on GENCOs' profit maximization using a new technique Binary Artificial Bee Colony algorithm based on global best parameters $(G b B A B C)$. The proposed approach is modeled as a bi-level optimization problem - the upper subproblem representing GENCOs' profit maximization and lower sub problem represents market clearing price. Optimal rival bidding strategy is employed to maximize GENCOs' profit. The rival's bidding behavior is estimated by Monte Carlo (MC) simulation and solved using (GbBABC). In the proposed algorithm, global best parameter was incorporated into $\mathrm{BABC}$ algorithm, which makes the exploitation capacity improved and convergence speed quickened. At the same time, in order to maintain the population diversity the bit mutation operator is also performed. The proposed algorithm has proper control on local optimum and global optimum, so that it can performs consistently and efficiently improves optimum solutions in the search space. Feasibility and robustness of the proposed method is tested on IEEE-30 and IEEE-57 bus system. From the results obtained demonstrated that BSO-NDC out performs for solving the proposed optimization problem in terms of solution quality, accuracy, computation time and convergence speed compared to other reported techniques such as $\mathrm{ABC}, \mathrm{Gb} \mathrm{ABC}$ and $\mathrm{Gbd} \mathrm{ABC}$.

\section{REFERENCES}

[1] David, A.K., and Wen, F., 2000. Strategic bidding in competitive electricity markets: a literature survey. IEEE PES Summer Meeting, vol.4, pp- 2168-2173.

[2] Li, T., and Shahidehpour, M., 2005. Strategic bidding of transmission-constrained GENCOs with incomplete information. IEEE Trans. Power Syst., vol.20, pp. 437447.

[3] Ferrero, R.W., Ramesh, V.C., and Shahidehpour, S.M., 1997. Transaction analysis in deregulated power system using game theory. IEEE Trans. Power Syst., vol. 12, pp. $1340-1347$.

[4] Torre, S.D., Conejo, A.J., and Contreras, J., 2004. Finding multi-period Nash equilibrium in pool-based electricity markets. IEEE Trans. Power Syst., vol.19, pp. 643-651.
[5] Bajpai, P., Punna, S.K., and Singh. S.N., 2008. Swarm intelligence-based strategic bidding in competitive electricity markets. IET Gener. Trans. Distr., vol. 2, pp.175-184.

[6] Jain, A.K., and Srivastava, S.C., 2009. Strategic Bidding and risk assessment using genetic algorithm in electricity markets. Intern. Journ of Emerging Elect Power Syst., vol.10, pp.1-10..

[7] Gountis, V.P., and Bakirtzis, A.G., 2004. Bidding strategies for electricity producers in a competitive electricity marketplace. IEEE Trans. Power Syst., vol.19, pp. 356-365.

[8] Soleymani, S., 2011 Bidding strategy of generation companies using PSO combined with SA method in the pay as bid markets. Intern. Journ of Electr Power Energy Syst., vol. 33, pp.1272-8.

[9] Peng, Ch., Huijuan, S., Jianfeng, G., and Liu. G., 2012. Multi-objective optimal strategy for generating and bidding in the power market. Energy Conv and management, vol. 57, pp.13-22

[10] Bajpai, P., and Singh. S.N., 2007. Fuzzy adaptive particle swarm optimization for bidding strategy in uniform price spot market. IEEE Trans Power Syst., vol.22, pp.215260 .

[11] Karaboga, D., and Basturk, B., 2008. On the performance of artificial bee colony (ABC) algorithm. Applied Soft Computing, vol.8, pp.687-697.

[12] Karaboga, N., 2009. A new design method based on artificial bee colony algorithm for digital IIR filters. Journal of the Franklin Institute, vol. 346, pp.328-348.

[13] Basu, M., 2013. Artificial bee colony optimization for multi-area economic dispatch. International Journal of Electrical Power \& Energy Syst., vol.49, pp.181-187.

[14] Singh, A., 2009. An artificial bee colony algorithm for the leaf constrained minimum spanning tree problem. Applied Soft Computing, vol.9, pp.625-631.

[15] Tasgetiren, M.F., Pan, Q.K., Suganthan, P.N., and Chen, A.H.L., 2011. A discrete artificial bee colony algorithm for the total flow time minimization in permutation flow shops. Information Sciences, vol.181, pp. 3459-3475.

[16] Ma, M., Lieang, J., Guo, M., Fan, Y., and Yin, Y., 2011. SAR image segmentation based on artificial bee colony algorithm. Applied Soft Computing, vol.11, pp.52055214.

[17] Huang, S. J., and Liu, X. Z., 2013. Application of artificial bee colony-based optimization for fault section estimation in power systems. Intern Journal of Electrical Power \& Energy Syst., vol.44, pp.210-218.

[18] Liao, X., Jianzhong, Z., Rui, Z., and Yongchuan, Z., 2012. An adaptive artificial bee colony algorithm for long-term economic dispatch in cascaded hydropower systems. Intern Journal of Electrical Power \& Energy Syst., vol. 43, pp.1340-1345.

[19] Chandrasekaran, K., Hemamalini, S., Sishaj, P., Simon \& Narayana, P. P., 2012. Thermal unit commitment using binary/real coded artificial bee colony algorithm. Electric Power Syst Research, vol.84, pp.109-119. 
[20] Li, Y., Wang, Y., and Bin, L., 2013. A hybrid artificial bee colony assisted differential evolution algorithm for optimal reactive power flow. Intern Journal of Electrical Power \& Energy Syst., vol. 52, pp. 25-33.

[21] Ozyon, S., and Aydin, D., 2013. Incremental artificial bee colony with local search to economic dispatch problem with ramp rate limits and prohibited operating zones. Energy Conversion and Management, vol.65, pp. 397-407.

[22] Karaboga, D., Ozturk, C., Karaboga, N., and Gorkemli, B., 2012. Artificial bee colony programming for symbolic regression. Information Sciences, vol. 209, pp.1-15.

[23] Alatas, B., 2010. Chaotic bee colony algorithms for global numerical optimization. Expert Systems with Applications. vol. 37, pp. 5682-5687.

[24] Gao, W., and Liu, S., 2012. A modified artificial bee colony algorithm. Computers and Operations Research, vol. 39, pp. 687-697,
[25] Zhu, G.., and Kwong, S., 2010. Gbest-guided artificial bee colony algorithm for numerical function optimization. Applied Mathematics and Computation, vol. 217 , no. 7, pp. 3166-3173.

[26] Banharnsakun, A., Achalakul, T., and Sirinaovakul, B. 2011. The best so- far selection in artificial bee colony algorithm. Applied Soft Computing, vol. 11, pp. 28882901.

[27] Kashan, M. H., Nahavandi, N., and Kashan, A.H., 2012. DisABC: A new artificial bee colony algorithm for binary optimization. Applied Soft Computing, vol.12, pp. 342-352.

[28] Andrej, A., Scheidler, S., Middendorf, M., and Diwold K., 2011. Performance evaluation of artificial bee colony optimization and new selection schemes. Memetic Computing, vol.3. pp. 149-162.

[29] http://www.ee.washington.edu./research/pstca 